\title{
New Method of Computing $\pi$ value (Siva Method)
}

\section{RD Sarva Jagannada Reddy}

\section{Introduction}

$\pi$ equal to $3.1415926 \ldots$ is an approximation. It has ruled the world for 2240 years. There is a necessity to find out the exact value in the place of this approximate value. The following method givesthe total area of the square, and also the total area of the inscribed circle. $\pi$ derived from this area is thus exact.

\section{Construction procedure}

Draw a circle with center ' 0 ' and radius $a / 2$. Diameter is ' $\mathrm{a}$ '. Draw 4 equidistant tangents on the circle. They intersect at $\mathrm{A}, \mathrm{B}, \mathrm{C}$ and $\mathrm{D}$ resulting in $\mathrm{ABCD}$ square. The side of the square is also equal to diameter ' $a$ '. Draw two diagonals. E, F, G and $\mathrm{H}$ are the mid points of four sides. Join EG, FH, EF, FG, GH and HE. Draw four arcs with radius $a / 2$ and with centres $\mathrm{A}, \mathrm{B}, \mathrm{C}$ and D. Now the circle square composite system is divided into 32 segments and number them 1 to 32.1 to 16 are of one dimension called $\mathrm{S}_{1}$ segments and 17 to 32 are of different dimension called $S_{2}$ segments.

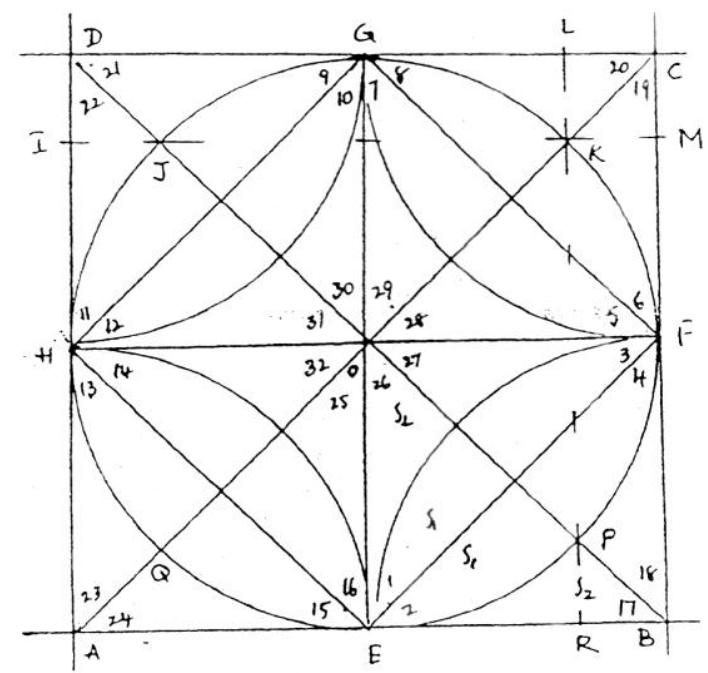

III. Calculations:

$\mathrm{ABCD}=$ Square Side $=\mathrm{a}, \mathrm{EFGH}=$ Circle, diameter $=\mathrm{a}$, radius $=\mathrm{a} / 2$

Area of the $\mathrm{S}_{1}$ segment $=\left(\frac{6-\sqrt{2}}{128}\right) a^{2} ;$ Area of the $\mathrm{S}_{2}$ segment $=\left(\frac{2+\sqrt{2}}{128}\right) a^{2}$;

Area of the square $=16 \mathrm{~S}_{1}+16 \mathrm{~S}_{2}=16\left(\frac{6-\sqrt{2}}{128}\right) a^{2}+16\left(\frac{2+\sqrt{2}}{128}\right) a^{2}=a^{2}$

Area of the inscribed circle $=16 \mathrm{~S}_{1}+8 \mathrm{~S}_{2}=16\left(\frac{6-\sqrt{2}}{128}\right) a^{2}+8\left(\frac{2+\sqrt{2}}{128}\right) a^{2}=\left(\frac{14-\sqrt{2}}{16}\right) a^{2}$

General formula for the area of the circle $\frac{\pi d^{2}}{4}=\frac{\pi a^{2}}{4}=\left(\frac{14-\sqrt{2}}{16}\right) a^{2}$; where $\mathrm{a}=\mathrm{d}=$ side $=$ diameter

$$
\therefore \pi=\frac{14-\sqrt{2}}{4}
$$


IV. How two formulae for $S_{1}$ and $S_{2}$ segments are derived ?

$16 \mathrm{~S}_{1}+16 \mathrm{~S}_{2}=\mathrm{a}^{2}=$ area of the Square $\quad$... Eq. (1)

$16 \mathrm{~S}_{1}+8 \mathrm{~S}_{2}=\frac{\pi a^{2}}{4}=$ area of the Circle $\quad \ldots$ Eq. (2)

$(1)-(2) \Rightarrow \quad 8 \mathrm{~S}_{2}=a^{2}-\frac{\pi a^{2}}{4}=\frac{4 a^{2}-\pi a^{2}}{4}=\mathrm{S}_{2}=\frac{(4-\pi) a^{2}}{32}=\frac{a^{2}}{32}(4-\pi)$

(2)x $2 \Rightarrow 32 \mathrm{~S}_{1}+16 \mathrm{~S}_{2}=\frac{2 \pi a^{2}}{4}$

$16 S_{1}+16 S_{2}=a^{2}$

... Eq. (1)

(3) - (1) $16 \mathrm{~S}_{1}=\frac{\pi a^{2}}{2}-a^{2} \quad=\mathrm{S}_{1}=\frac{a^{2}(\pi-2)}{32}=\frac{a^{2}}{32}(\pi-2)$

V. Both the $\pi$ values appear correct when involved in the two formulae

a) Official $\pi$ value $=3.1415926 \ldots$

b) Proposed $\pi$ value $=3.1464466 \ldots=\frac{14-\sqrt{2}}{4}$

Hence, another approach is followed here to decide real $\pi$ value.

\section{Involvement of line-segments are chosen to decide real $\pi$ value.}

A line-segment equal to the value of $(\pi-2)$ in $S_{1}$ segment's formula and second line-segment equal to the value of $(4-\pi)$ in $S_{2}$ segment's formula are searched in the above construction.

a) Official $\pi: \pi-2=3.1415926 \ldots-2=1.1415926 \ldots$.

Proposed $\pi: \pi-2=\frac{14-\sqrt{2}}{4}-2=\frac{6-\sqrt{2}}{4}$

The following calculation gives a line-segment for $\frac{6-\sqrt{2}}{4}$ and no line-segment for 1.1415926. .

$\mathrm{IM}$ and LR two parallel lines to $\mathrm{DC}$ and $\mathrm{CB} ; \mathrm{OK}=\mathrm{OJ}=$ Radius $=\frac{a}{2} ; \mathrm{JOK}=$ triangle

$\mathrm{JK}=$ Hypotenuse $=\frac{\sqrt{2} a}{2}$

Third square $=$ LKMC; $\quad \mathrm{KM}=\mathrm{CM}=$ Side $=$ ?

$\mathrm{KM}=\frac{I M-J K}{2}=\left(a-\frac{\sqrt{2} a}{2}\right) \frac{1}{2}=\left(\frac{2-\sqrt{2}}{4}\right) a ; \quad$ Side of first square $\mathrm{DC}=\mathrm{a}$

$\mathrm{DC}+\mathrm{CM}=a+\left(\frac{2-\sqrt{2}}{4}\right) a=\left(\frac{6-\sqrt{2}}{4}\right) a$

b) Official $\pi=4-\pi=4-3.1415926 \ldots=0.8584074 \ldots$.

Proposed $\pi=4-\pi=4-\frac{14-\sqrt{2}}{4}=\frac{2+\sqrt{2}}{4}$

No line-segment for $0.8584074 \ldots$ in this diagram.

MB line-segment is equal to $\frac{2+\sqrt{2}}{4}$. How ?

Side of the first square $\mathrm{CB}=\mathrm{a}$

$\mathrm{MB}=\mathrm{CB}-\mathrm{CM}=a-\left(\frac{2-\sqrt{2}}{4}\right) a=\left(\frac{2+\sqrt{2}}{4}\right) a$ 


\section{Conclusion:}

This diagram not only gives two formulae for the areas of $S_{1} \& S_{2}$ segments andalso shows two linesegments for $(\pi-2)$ and $(4-\pi)$. Line-segment is the soul of Geometry. 